\title{
Strength of the Solar Coronal Magnetic field - A Comparison of Independent Estimates Using Contemporaneous Radio and Whitelight Observations
}

\author{
Anshu Kumari ${ }^{1}$. R. Ramesh ${ }^{1}$. \\ C. Kathiravan ${ }^{1}$. T. J. Wang ${ }^{2}$ \\ (C) Springer $\bullet \bullet \bullet$
}

\begin{abstract}
We estimated the coronal magnetic field strength $(B)$ during the 23 July 2016 coronal mass ejection (CME) event using i) the flux rope structure of the CME in the whitelight coronagraph images and ii) the band splitting in the associated type II burst. No models were assumed for the coronal electron density $(N(r))$ used in the estimation. The results obtained using the above two independent methods correspond to different heliocentric distances $(r)$ in the range $\approx 2.5-$ $4.5 \mathrm{R}_{\odot}$, but they show excellent consistency and could be fitted with a single power-law distribution of the type $B(r)=5.7 r^{-2.6} \mathrm{G}$, which is applicable in the aforementioned distance range. The power law index (i.e. -2.6$)$ is in good agreement with the results obtained in previous studies by different methods.
\end{abstract}

Keywords: Sun; Coronal Mass Ejections; Radio Bursts; Coronal magnetic field

\section{Introduction}

Estimation of the magnetic field strength $(B)$ in the solar corona is one of the widely pursued areas of research in observational solar physics. Circular

\footnotetext{
$凶$ Anshu Kumari

anshu@iiap.res.in

$\triangle$ R. Ramesh ramesh@iiap.res.in

$凶$ C. Kathiravan kathir@iiap.res.in

$\triangle$ T. J. Wang tongjiang.wang@nasa.gov

1 Indian Institute of Astrophysics, 2nd Block, Koramangala, Bangalore-560034, India

2 Department of Physics, The Catholic University of America and NASA Goddard Space Flight Center, Code 671, Greenbelt, MD 20771, USA
} 
polarization observations in the radio frequency range $1-20 \mathrm{GHz}$ have been extensively used to measure $B$ above active regions. The corresponding heliocentric distance range is typically $r \approx 1.05-1.10 \mathrm{R}_{\odot}$ (see for example Gelfreikh, 2004; Ryabov, 2004; White, 2004; and the references therein). Observations of Stokes $V$ profiles of the coronal emission line Fe XIII $\lambda 10747$ resulting from the longitudinal Zeeman effect were used by Lin, Penn, and Tomczyk (2000) to estimate $B$ over $r \approx 1.12-1.15 \mathrm{R}_{\odot}$. The above measurements in the radio and optical/infrared regimes are limited to the inner corona $\left(r \lesssim 1.2 \mathrm{R}_{\odot}\right)$. Moving over to the outer corona $\left(r>3 \mathrm{R}_{\odot}\right)$, Faraday rotation observations are generally used to derive the magnetic field (Patzold et al., 1987; Spangler, 2005; Mancuso and Garzelli, 2013). Of late, the standoff distance of a CME driven shock has also been used to estimate $B$ in the range $r \approx 3-15 \mathrm{R}_{\odot}$ (Kim et al., 2012), and $r \approx 60-215 \mathrm{R}_{\odot}$ (Poomvises et al., 2012). Compared to the above two regions of the corona, estimates of $B$ in the middle corona $\left(r \approx 1.2-3.0 \mathrm{R}_{\odot}\right)$ are primarily using the different types of transient non-thermal radio emission observed at low frequencies $(\lesssim 150 \mathrm{MHz})$ with ground based instruments. The radio methods, though indirect in contrast to the Zeeman effect in optical wavelengths, have provided the bulk of the quantitative information on $B$ in the above distance range (see Dulk and McLean, 1978 for a review on the topic). The various related techniques employed presently are based on band splitting in type II bursts, second harmonic plasma emission in type II, type III, and type IV bursts, gyrosynchrotron emission in type IV bursts, Alfv́en speed in type II bursts, quasi-periodicity in type III bursts, etc. Note that while the mechanism is either fundamental and/or second harmonic plasma emission in the case of type II and type III bursts, it is either second harmonic plasma or gyrosynchrotron emission in the case of type IV bursts. We would like to point out here that characteristics of the polarization of fundamental plasma emission from solar radio bursts is yet to be established. So, estimates of $B$ are currently possible only using second harmonic plasma emission from the bursts. The estimates are from observations of either the total intensity, i.e. Stokes I, alone (Smerd, Sheridan, and Stewart, 1975; Gopalswamy and Kundu, 1990; Bastian et al., 2001; Vršnak et al., 2002; Mancuso et al., 2003; Ramesh et al., 2003; 2004; 2013; Cho et al., 2007; Kishore et al., 2016) or both the total and circularly polarized intensities, i.e. Stokes $I$ and $V$ (Dulk and Suzuki, 1980; Gary et al., 1985; Ramesh et al., 2010a; Ramesh, Kathiravan, and Narayanan,2011; Tun and Vourlidas,2013; Sasikumar Raja and Ramesh, 2013; 2014; Hariharan et al., 2014; 2016b; Anshu et al., 2017). Note that we have mentioned only Stokes $I$ and $V$ emission here since differential Faraday rotation of the plane of polarization in the solar corona and Earth's ionosphere makes it impossible to observe the linear polarization (represented by Stokes $Q$ and $U$ ) within the typical observing bandwidths of $\approx 100 \mathrm{kHz}$ (see for example Grognard and McLean, 1973.) It has been shown that thermal radio emission observed from the solar corona at low frequencies can also be used to estimate $B$ (Ramesh, Kathiravan, and Sastry, 2003; Sastry, 2009; Ramesh, Kathiravan, and Sastry, 2010).

Gopalswamy et al. (2012) reported a new technique, based on the geometrical properties of the flux rope structure of a whitelight CME and the associated 
shock speed, to estimate $B$. Similarly, Kwon et al. (2013b; 2013a) reported estimates of $B$ from measurements of the propagation speed of a fast magnetosonic wave associated with a CME. But, reports of comparison between the $B$ values obtained independently in radio and optical wavelengths for the same event using simultaneous observations which do not assume any model for $N(r)$ are rare. The comparison is important to validate the different techniques to estimate $B$. Considering this, in the present work we take advantage of the simultaneous observations of a whitelight CME near the Sun with flux rope structure and band splitting exhibited by the associated type II burst, we estimate the magnetic field strength in the middle corona.

\section{Observations}

\subsection{Radio Observations}

The radio data were obtained with the Gauribidanur RAdio SpectroPolarimeter (GRASP; Kishore et al., 2014, Hariharan et al., 2016a) with a new digital backend correlator receiver, operated at the Gauribidanur radio observatory 11 about $100 \mathrm{~km}$ north of Bangalore in India (Ramesh, 2011). The primary receiving elements of GRASP are two log-periodic dipole antennas (LPDAs) oriented orthogonal to each other. The characteristics of these LPDAs are: half-power beam width $(\mathrm{HPBW}) \approx 100^{\circ}$ (in both the $\mathrm{E}$ - and $\mathrm{H}$-planes), gain $\mathrm{G} \approx 5.5 \mathrm{~dB} 2$ and effective collecting area $\mathrm{A}_{\mathrm{e}} \approx 0.3 \lambda^{2}$. The radio frequency $(\mathrm{RF})$ signals from the two LPDAs are transmitted to the receiver room using independent low loss coaxial cables where a 2-channel, 8-bit Analog-to-Digital Converter (ADC) is used to directly digitize the RF signal from each LPDA. The maximum possible sampling clock for the ADC is $\approx 250 \mathrm{MHz}$. Since the RF signals received by the LPDAs in the GRASP are limited to $\lesssim 100 \mathrm{MHz}$, they were sampled at $200 \mathrm{MHz}$. The ADC was interfaced to a $\mathrm{ROACH}^{3}$ board. The signals from the two channels of the $\operatorname{ADC}\left(E_{x}\right.$ and $E_{y}$, respectively) were Fourier transformed to obtain the instantaneous spectrum with a spectral resolution of $\approx 200 \mathrm{kHz}$ and temporal resolution of $\approx 100 \mathrm{msec}$. The spectrum thus obtained is used to compute Stokes $I$ and $V$ intensities as follows (Collett, 1992):

$$
\begin{gathered}
I=<E_{x} E_{x}^{*}+E_{y} E_{y}^{*}> \\
V=-2 i<E_{x} E_{y}^{*}-E_{y} E_{x}^{*}>
\end{gathered}
$$

Figure 1shows the dynamic spectrum of the transient emission from the Sun observed with the GRASP on 23 July 2016. The fast drifting feature (from high to low frequencies) at $\approx 05: 30 \mathrm{UT}$ is a type $\mathrm{V}$ burst. The slow drifting feature

\footnotetext{
${ }^{1}$ See http://www.iiap.res.in/centers/radio

${ }^{2}$ The gain of an antenna system relative to an isotropic radiator.

${ }^{3}$ See https://casper.berkeley.edu/.
} 


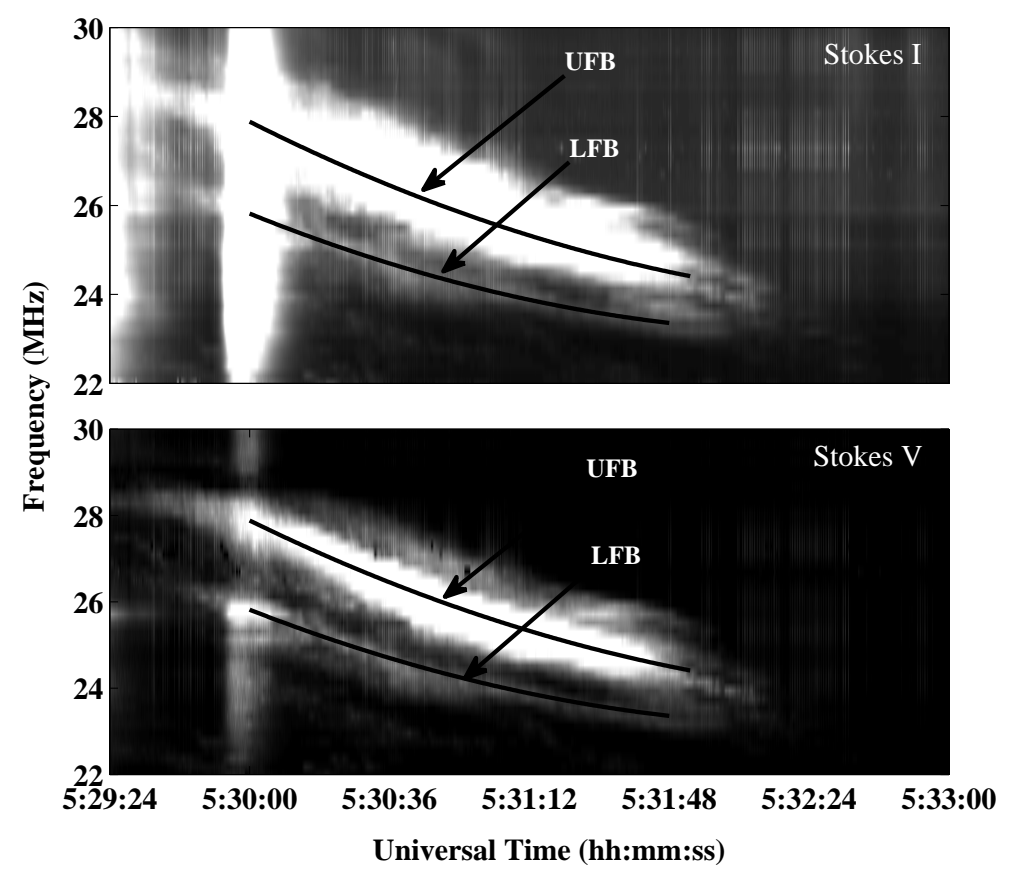

Figure 1 . Type V burst $(\approx 05: 30 \mathrm{UT})$ and type II radio burst $(\approx 05: 30-05: 32 \mathrm{UT})$ observed with the GRASP on 23 July 2016. The band-splitting of the type II burst can be clearly noticed in both the Stokes $I$ and $V$ spectra. The black lines indicate the upper and lower frequency bands of the split-band emission. The thin, closely spaced vertical lines seen in the spectra are artefacts.

between $\approx 05: 30-05: 32$ UT is a type II burst. While the type II burst is confined to a narrow range of frequencies (start frequency $\approx 28 \mathrm{MHz}$; end frequency $\approx 24 \mathrm{MHz}$ ), the type $\mathrm{V}$ burst extends beyond. We verified the aforementioned frequency limits from observations with the Gauribidanur LOw-frequency Solar Spectrograph (GLOSS; Ebenezer et al., 2001; 2007; Kishore et al., 2015) and the e-CALLISTO (Monstein, Ramesh, and Kathiravan, 2007; Benz et al., 2009) in the frequency range $\approx 40-450 \mathrm{MHz}$. Note that observations of type II bursts over a limited spectral range as in the present work are not uncommon (see for example Hariharan, Ramesh, and Kathiravan, 2015). We will discuss the above type II burst in the rest of this work.

Solar type II bursts are signatures of magnetohydrodynamic (MHD) shock waves travelling outwards in the corona. These shocks accelerate electrons to suprathermal velocities. The energetic electrons excite Langmuir (plasma) waves which are converted into radio waves escaping the corona (see Roberts, 1959; Wild, Smerd, and Weiss, 1963; Nelson and Melrose, 1985; Mann, Classen, and Aurass, 1995; Aurass, 1997; Gopalswamy, 2006 for details). A closer inspection of the type II burst in Figure 1indicates that it is split, and appears as two bands 


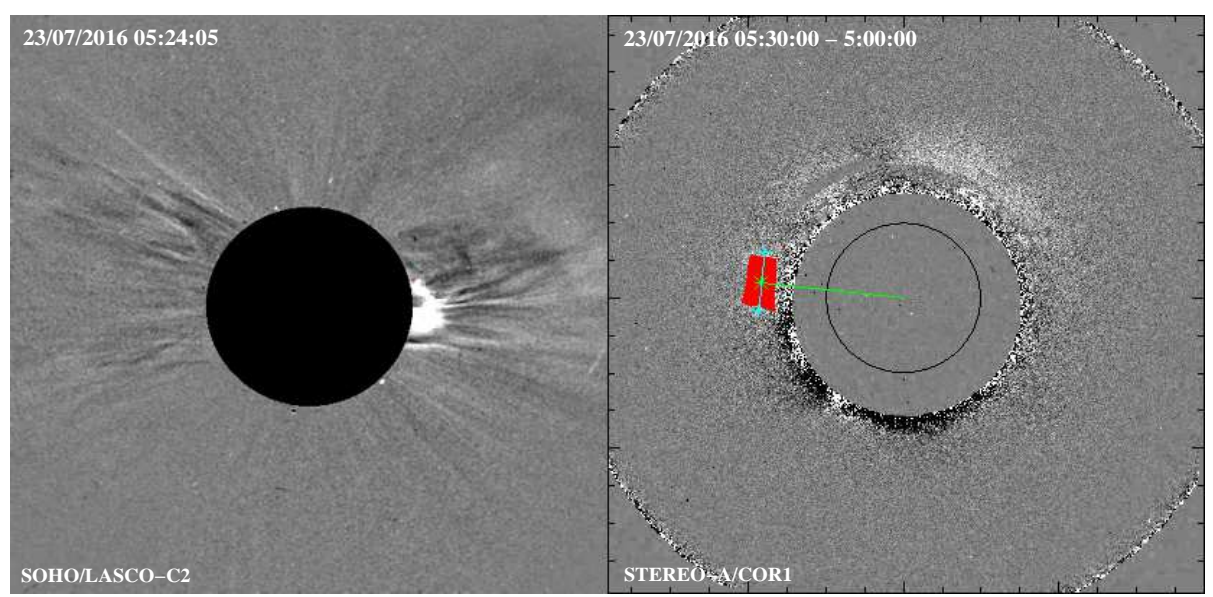

Figure 2. Left Panel: $\mathrm{SOHO} / \mathrm{LASCO}-\mathrm{C} 2$ coronagraph difference image of the CME that occurred on 23 July 2016 around $\approx 05: 24$ UT. The black circle (radius $\approx 2.2 \mathrm{R}_{\odot}$ ) represents the occulting disk of the coronagraph. Solar north is straight up and east is to the left in the image. Right Panel: STEREO-A/COR1 pB difference image of the same CME as in the left panel. The black and the gray circles indicate the solar limb (radius $=1 \mathrm{R}_{\odot}$ ) and the coronagraph occulter (radius $\approx 1.4 \mathrm{R} \odot$ ), respectively. The CME is fainter compared to the $\mathrm{SOHO} / \mathrm{LASCO}-\mathrm{C} 2$ observations since its angle with respect to the plane-of-sky (POS) is relatively larger for STEREO-A (see Section 2.2 for details). The electron density of the CME mentioned in Section 3.1 was estimated from the coronal region covered by the red rectangular box. The vertical line across the above box represents the width of the CME $\left(\approx 0.79 \mathrm{R}_{\odot}\right)$.

(upper frequency band [UFB] and lower frequency band [LFB]). The frequency ranges are $\approx 28.0-24.2 \mathrm{MHz}(\mathrm{UFB})$ and $\approx 26.0-23.5 \mathrm{MHz}(\mathrm{LFB})$. The LFB and UFB bands relate to emission from the coronal regions ahead of and behind the associated MHD shock, respectively (Vršnak, 2001).

\subsection{STEREO-A/COR1 and SOHO/LASCO-C2 Observations}

The optical data were obtained with the Large Angle and Spectrometric Coronagraph C2 (LASCO-C2; Brueckner et al., 1995) on board the Solar and Heliospheric Observatory (SOHO), and the COR1 coronagraph of the Sun-Earth Connection Coronal and Heliospheric Investigation (SEECHI; Howard et al., 2008) on board the Solar Terrestrial Relationship Observatory (STEREO). The type II burst in Figure 1 was associated with a whitelight CME observed by the SOHO/LASCO-C2 and STEREO-A/COR1 coronagraphs. The CME was noticed in the SOHO/LASCO-C2 field-of-view (FOV) around $\approx 05: 24$ UT (see left panel in Figure2). Its central position angle (PA, measured counter-clockwise from the solar north) during the above epoch is $\approx 267^{\circ}$, and the angular width is $\approx 65^{\circ}$. The projected heliocentric distance of the $\mathrm{CME}$ is $\approx 2.79 \mathrm{R} \odot$. 4 . There

${ }^{4}$ See http://spaceweather.gmu.edu/seeds/monthly.php? $\mathrm{a}=2016 \mathrm{k} b \mathrm{~b}=07$. 
was a 3B class $\mathrm{H} \alpha$ flare from AR12565 located on the solar disk at heliographic coordinates N02W75. The flare was observed during the interval $\approx 05: 09-06: 33$ UT. The peak was at $\approx 05: 31$ UT5. The X-Ray Sensor (XRS) on board the Geostationary Operational Environmental Satellite (GOES-15) observed a M5.5 class soft X-ray flare from the above active region, during the interval $\approx 05: 27$ 05:33 UT. The peak time was the same as that of the $\mathrm{H} \alpha$ flare, i.e. $\approx 05: 31 \mathrm{UT}$. This indicates that the onset of the type II burst $(\approx 05: 30 \mathrm{UT})$ in Figure 1 was close to the peak time of the flare.

The right panel in Figure 2 shows the STEREO-A/COR1 difference image of the solar corona obtained on 23 July 2016 at $\approx 05: 30 \mathrm{UT}$. The boxed region at PA $\approx 84^{\circ}$ indicates the same CME noticed in the SOHO/LASCO-C2 observations in the left panel of Figure 2 at $\approx 5: 24$ UT. Its projected heliocentric distance in the STEREO-A/COR1 FOV is $\approx 1.86 \mathrm{R}_{\odot}$. The subtracted reference image for STEREO-A/COR1 in Figure 2 was observed at $\approx 05: 00$ UT. We found that STEREO-A was at $\approx E 153^{\circ}$ during the above observation. 6 . Therefore for the STEREO-A view, the flaring region is on the far side of the Sun at $\approx 42^{\circ}$ behind the east limb. For the SOHO/LASCO-C2 view, the above region is located on the solar disk at $\approx 15^{\circ}$ from the west limb. The CME observations will have projection effects in view of the above disk locations. We calculated the deprojected heliocentric distances of the CME from the SOHO/LASCO-C2 and STEREO-A/COR1 images assuming that the projection effects vary as $1 / \cos (\phi)$, where $\phi$ is the angle from the plane-of-sky (POS).

\section{Analysis and Results}

\subsection{Estimates of $N(r)$ Using Polarized Brightness (pB) Measurements with STEREO-A/COR1 and SOHO/LASCO-C2}

The electron density in the corona depends upon the brightness and the polarization in the corona (van de Hulst, 1950). We used polarized Brightness $(\mathrm{pB})$ measurements with the STEREO-A/COR1 and SOHO/LASCO-C2 coronagraphs (see Figure 21) to find a suitable model for $N(r)$ to estimate the $B(r)$ values. In the case of STEREO-A/COR1, the $N(r)$ of the background corona and the CME were calculated using the images obtained at $\approx 05: 00 \mathrm{UT}$ (i.e. before the onset of the $\mathrm{CME}$ ) and $\approx 05: 30$ UT (during the type II burst), respectively. These calculations were done using the spherically symmetric inversion technique (Wang and Davila, 2014). The $N(r)$ of the background corona was calculated in the distance range $r \approx 1.5-3.7 \mathrm{R}_{\odot}$ and over $\mathrm{PA} \approx 84^{\circ} \pm 5^{\circ}$ (see right panel in Figure 21). In case of the CME, the background subtracted $\mathrm{pB}$ radiation from the boxed region in the right panel of Figure 2 was used. The de-projected heliocentric distance of the latter is $\approx 2.63 \mathrm{R}_{\odot}$. We assumed that the line-of-sight (LOS) depth of the CME is equal to its width, and the CME

${ }^{5}$ See ftp://ftp.ngdc.noaa.gov/STP/swpc_products/daily_reports/solar_event_reports/2016/07.

${ }^{6}$ See stereo-ssc.nascom.nasa.gov/cgi-bin/make_where.gif. 


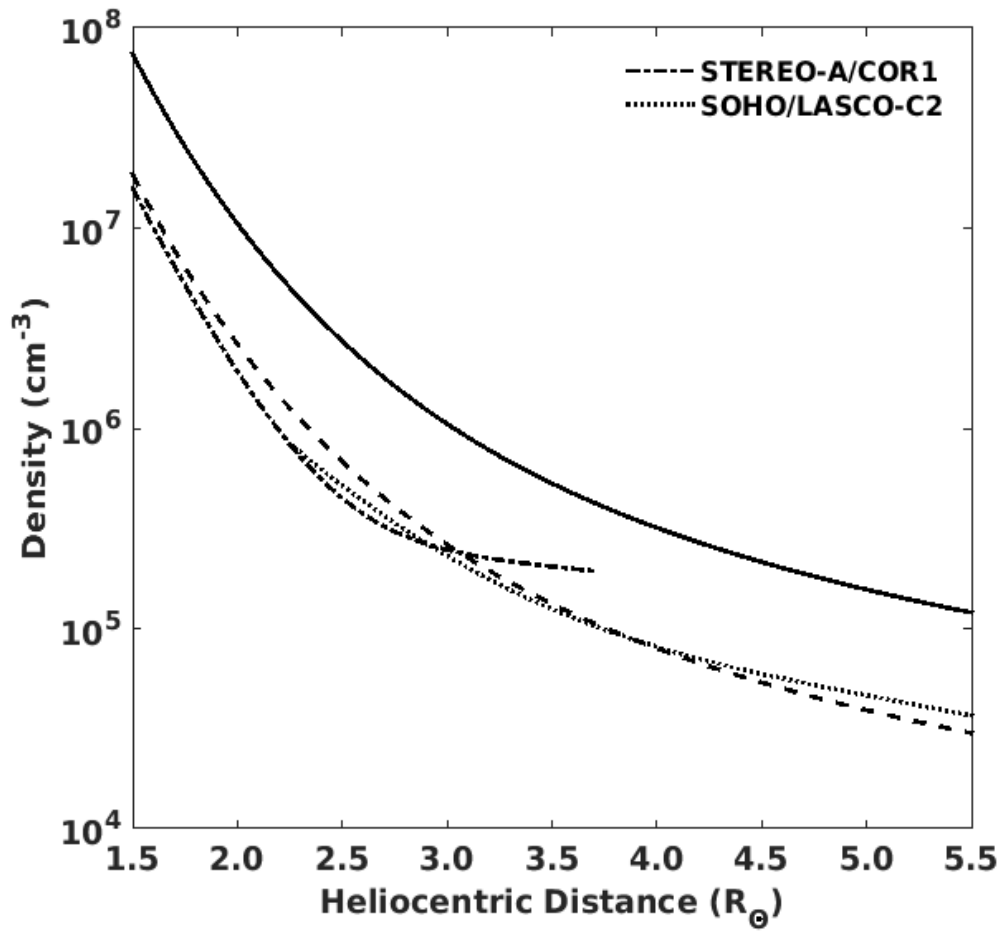

Figure 3. Estimates of $N(r)$ in the background corona using pB measurements with STE$\mathrm{REO}-\mathrm{A} / \mathrm{COR} 1\left(r \approx 1.5-3.7 \mathrm{R}_{\odot}\right)$ and SOHO/LASCO-C2 $\left(r \approx 2.3-5.5 \mathrm{R}_{\odot}\right)$ corongraphs. The dashed line $\left(r \approx 1.5-5.5 \mathrm{R}_{\odot}\right)$ is the best fit to the measurements of $N(r)$ with the above two instruments. The solid line in the same distance range represents $4 \times$ the density values corresponding to the above fit.

is at an angle of $\approx 42^{\circ}$ relative to the POS (see Section 2.2 ) for the above calculations. The $N(r)$ of the background corona and CME is $\approx 0.37 \times 10^{6} \mathrm{~cm}^{-3}$ and $\approx(1.8 \pm 3.3) \times 10^{6} \mathrm{~cm}^{-3}$, respectively at $r \approx 2.63 \mathrm{R}_{\odot}$.

In the case of $\mathrm{SOHO} / \mathrm{LASCO}-\mathrm{C} 2, N(r)$ of the background corona was calculated over the range $\mathrm{PA} \approx 256^{\circ} \pm 5^{\circ}$ using the image obtained at $\approx 02: 54 \mathrm{UT}$ (see left panel in Figure 22). The distance range was $r \approx 2.3-5.5 \mathrm{R}_{\odot}$. At the deprojected heliocentric distance $\approx 2.63 \mathrm{R}_{\odot}$, the background density is $\approx 0.43 \times$ $10^{6} \mathrm{~cm}^{-3}$. Considering this along with the previously mentioned similar measurements with STEREO-A/COR1, the average $N(r)$ value in the background corona at the location of the CME $\left(r \approx 2.63 \mathrm{R}_{\odot}\right.$ ) is $\approx 0.4 \times 10^{6} \mathrm{~cm}^{-3}$ (see right panel in Figure 22). This implies the total $N(r)$ at the CME location is $\approx 2.2$ $\times 10^{6} \mathrm{~cm}^{-3}$ (CME plus background corona).

Figure 3 shows the background density estimates for STEREO-A/COR1 and $\mathrm{SOHO} / \mathrm{LASCO}-\mathrm{C} 2$ for the distance range $r \approx 1.5-3.7 \mathrm{R}_{\odot}$ and $r \approx 2.3-6.4 \mathrm{R}_{\odot}$, respectively. We found that the best fit to the background density is $N_{\text {cor }}(r)=$ $1.521 \times 10^{8} r^{-7.279}+1.84 \times 10^{8} r^{-7.938}+2.07 \times 10^{7} r^{-4.852}+7.52 \times 10^{5} r^{-2.024}$, where the first two terms correspond to $r \approx 1.5-2.5 \mathrm{R}_{\odot}$ and $r \approx 2.5-3.7 \mathrm{R}_{\odot}$ in 
the STEREO-A/COR1 FOV, and the last two terms correspond to $r \approx 2.3-$ $4.0 \mathrm{R}_{\odot}$ and $r \approx 4.0-5.5 \mathrm{R}_{\odot}$ in the $\mathrm{SOHO} / \mathrm{LASCO}-\mathrm{C} 2 \mathrm{FOV}$, respectively. Note that the widely used Saito model for $N(r)$, derived from similar corongraph observations in the range $r \approx 2.5-5.5 \mathrm{R}_{\odot}$ (Saito, Poland, and Munro, 1977), has also two different radial gradients as in the present case.

The plasma frequency $\left(f_{\mathrm{p}}\right)$ corresponding to the estimates of the total density $\left(\approx 2.2 \times 10^{6} \mathrm{~cm}^{-3}\right)$ is $\approx 13.3 \mathrm{MHz}$. This implies that the type II burst in Figure 1 is most likely due to second harmonic $\left(2 f_{\mathrm{p}}\right)$ emission since its mean frequency at $\approx 05: 30 \mathrm{UT}$ is $\approx 27 \mathrm{MHz}$. The estimated maximum degree of circular polarization dcp is $\approx 18 \%$ and average dcp is $\approx 14 \%$ of the type II burst, which are also consistent with that expected for harmonic plasma emission (Dulk and Suzuki, 1980). We would like to point out here that there are reports of third harmonic emission $\left(3 f_{\mathrm{p}}\right)$ of type II bursts also in the literature. But such observations are rare (Roberts, 1959; Bakunin et al., 1990: Kliem, Krueger, and Treumann, 1992; Zlotnik et al., 1998). Interestingly, GLOSS observed the third harmonic emission of the above type II event in the frequency range $\approx 56-43 \mathrm{MH} 7^{7}$. This was possible most likely because of the comparatively larger collecting area of GLOSS. Note that the latter consists of eight antennas for radio frequency signal reception as compared to the typical solar radio spectrographs where only a single antenna is used (see for example Benz et al., 2009). Further, Sun was closer to the local zenith in Gauribidanur during the observations. The antenna gain is always maximum in that direction of the sky.

\subsection{Speed of the CME}

The CME was noticed in the STEREO-A/COR1 FOV during $\approx 05: 25-05: 35$ UT. In the case of SOHO/LASCO-C2, it was in the interval $\approx 05: 24-06: 12$ UT. The deprojected height-time $(h-t)$ plot of the CME LE obtained from the above observations is shown in Figure 4 The simultaneous measurements of the CME LE location with the above two instruments during $\approx 05: 24-05: 36$ UT are reasonably consistent. This confirms that the SOHO/LASCO-C2 and STEREO-A/COR1 observations described above correspond to the same CME. Its estimated speed $\left(v_{\mathrm{CME}}\right)$ based on the linear-fit to the $(h-t)$ measurements in Figure 4 is $\approx 735 \mathrm{~km} / \mathrm{s}$.

\subsection{Estimates of $B(r)$}

\subsubsection{Radio Observations}

The pB measurements using STEREO-A/COR1 and SOHO/LASCO-C2 coronagraphs described in Section 3.1 indicate that $N(r) \approx 2.2 \times 10^{6} \mathrm{~cm}^{-3}$ (CME plus background corona) at $r \approx 2.63 \mathrm{R}_{\odot}$. This is $\approx 4 N_{\text {cor }}(r)$ of the background corona at the above location (see Figure 3). We used the UFB and LFB bands of the type II burst (marked with black lines in Figure 1) to calculate $B(r)$

${ }^{7}$ http://www.iiap.res.in/files/solarradioimages/gbd/GLOSS_20160723.jpg. 


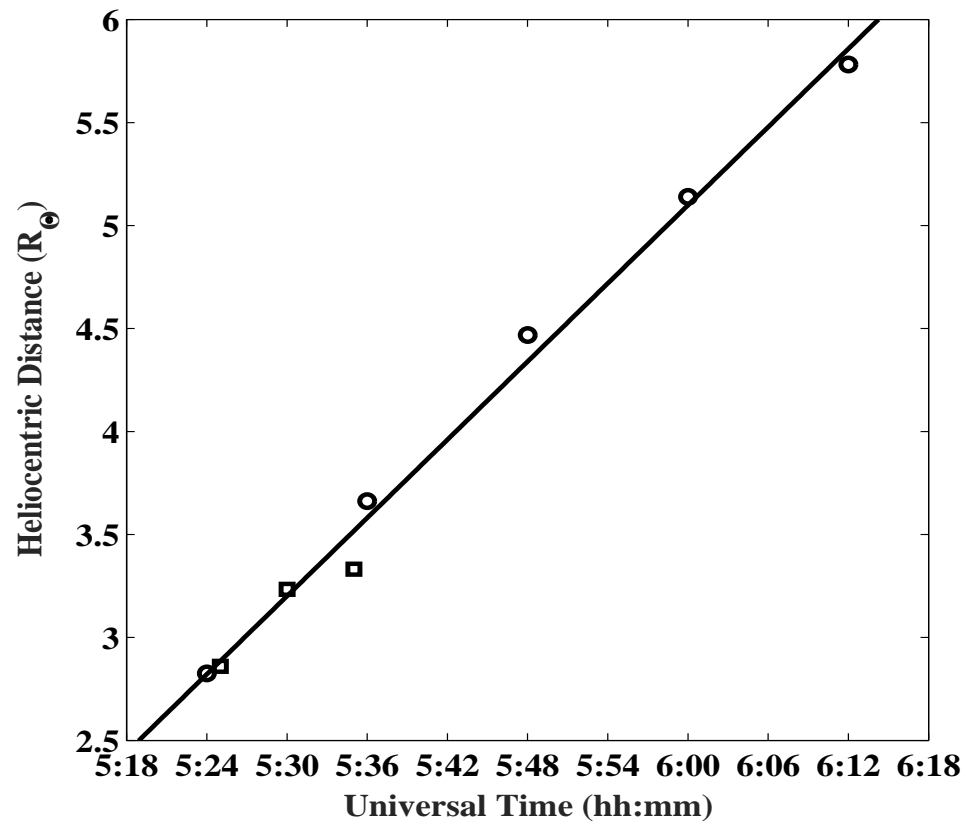

Figure 4. Height-time $(h-t)$ plot showing the de-projected heliocentric distances of the CME LE observed with the STEREO-A/COR1 coronagraph (indicated by squares) and SOHO/LASCO-C2 coronagraph (indicated by circles) on 23 July 2016 at different epochs. The solid line is the linear fit to the estimates.

using the split-band technique (see for example Vršnak et al., 2002; Cho et al., 2007). The drift rate of the burst is $\approx 0.35 \mathrm{MHz} / \mathrm{s}$. We converted this drift rate of the burst to shock speed $\left(v_{\mathrm{S}}\right)$ using the $4 N_{\text {cor }}(r)$ model mentioned earlier (see Section 3.1). The mean value of $v_{\mathrm{S}}$ for the four different spectra (i.e. LFB and UFB in Stokes $I$ and $V$ ) is $\approx 800 \mathrm{~km} / \mathrm{s}$. This is nearly same as the $v_{\mathrm{CME}}$ in Figure 4. implying the type II burst is most likely due to the MHD shock driven by the CME LE (Ramesh et al., 2010b; Ramesh et al., 2012). The low start frequency of the type II burst also indicates the same (Gopalswamy, 2006). We then calculated $B(r)$ using the following equation:

$$
B(G)=5.1 \times 10^{-5} v_{\mathrm{A}} f_{\mathrm{p}}
$$

where $v_{\mathrm{A}}$ is the Alfv́en speed. It was obtained using the parameters like instantaneous bandwidth, density jump, Alfv́enic Mach number $M_{\mathrm{A}}$ related to the split-band type burst in Figure1 (see Vršnak et al., 2002 for the relationship between the above parameters), and the relationship, $v_{\mathrm{A}}=v_{\mathrm{S}} / M_{\mathrm{A}}$.

The results indicate that $B(r) \approx(0.47-0.44) \pm 0.02 \mathrm{G}$ in the range $r \approx 2.61-$ $2.74 \mathrm{R}_{\odot}$. Note that the above distance interval corresponds to $f_{\mathrm{p}} \approx 14-12 \mathrm{MHz}$ (the fundamental component of the split-band type II burst in the present case, see Figure 1 and Section 2.2) since the $2 f_{\mathrm{p}}$ emission occurring at the same time 


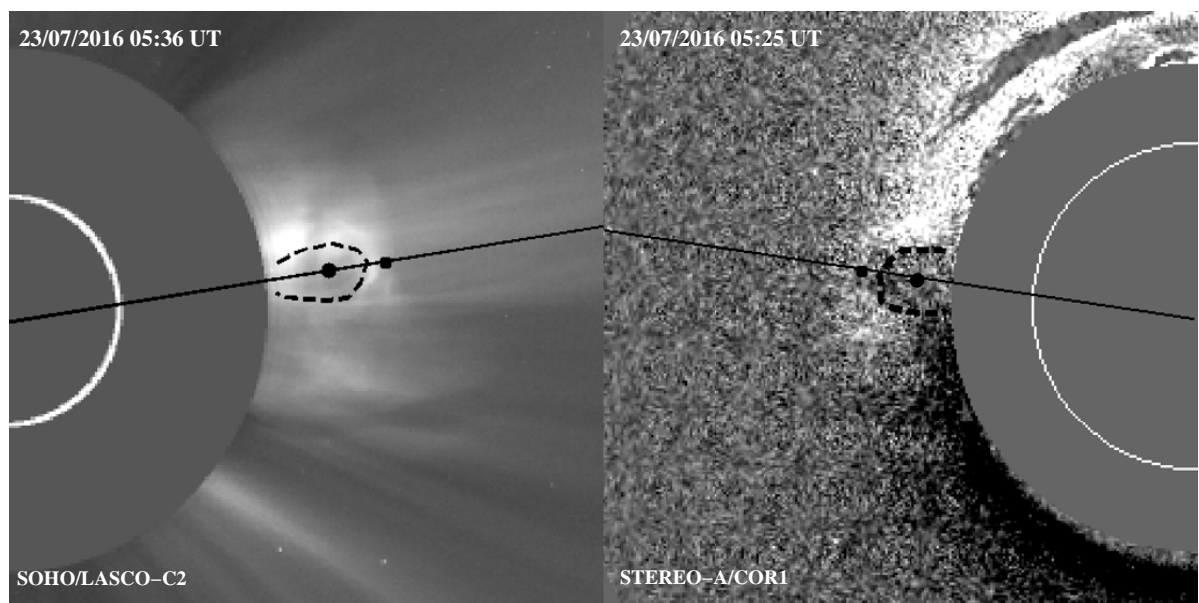

Figure 5. Left Panel: Same as the observations in the left panel of Figure2 but obtained at $\approx 05: 36 \mathrm{UT}$. The white circle indicates the solar limb (radius $\left.=1 \mathrm{R}_{\odot}\right)$. The different features of the CME used for the estimates of $B$ (see Table 1) are: black square - location of the CME shock, black dashed lines - CME flux rope, black dot - center of the circle fitted to the flux rope. Right Panel: Same as the observations in the right panel of Figure 2 but obtained at $\approx 05: 25 \mathrm{UT}$. The white circle indicates the solar limb (radius $=1 \mathrm{R} \odot$ ). The different features of the CME used for the estimates of $B$ (see Table 1) are: black square - location of the CME shock, black solid lines - CME flux rope, black dot - center of the circle fitted to the flux rope (see Gopalswamy et al., 2012 for details). The black solid line connects the aforementioned CME features with the center of the Sun.

as the $f_{\mathrm{p}}$ emission should be generated at the same location as the $f_{\mathrm{p}}$ emission (Smerd, Wild, and Sheridan, 1962).

\subsubsection{Whitelight Observations}

We also estimated $B(r)$ using the whitelight images obtained with STEREOA/COR1 (right panel in Figure 5) and SOHO/LASCO-C2 (left panel in 5), and the shock standoff distance technique described in Gopalswamy et al. (2012). The basic CME related parameters used in the above technique, i.e. the heliocentric distance of the CME flux rope $R_{\mathrm{ff}}$, leading edge of the shock $R_{\mathrm{sh}}$ and center of curvature of the flux rope $R_{\mathrm{c}}$ were measured for the aforesaid images as well as their next images observed with the respective coronagraphs. We assumed the adiabatic exponent $\gamma$ to be $4 / 3$ for the calculations. The stand-off distance $\Delta R$, $M_{\wedge}$ and $v_{\mathrm{s}}$ were calculated using the above parameters (see Gopalswamy et al. (2012) for the relationship between the above parameters), and $v_{\mathrm{A}}$ was estimated as earlier (Section 3.3.1). Adopting the same $4 N_{\text {cor }}(r)$ model as in the $B(r)$ estimate using the radio observations, we estimated the density for STEREO$\mathrm{A} / \mathrm{COR} 1$ and $\mathrm{SOHO} / \mathrm{LASCO}-\mathrm{C} 2$ at $r \approx 3.11 \mathrm{R}_{\odot}$ and $r \approx 4.40 \mathrm{R}_{\odot}$, respectively. The magnetic field strength in the corona was estimated using Equation 3, and the values are $B(r) \approx 0.30 \mathrm{G}$ at $r \approx 3.11 \mathrm{R}_{\odot}$ (STEREO-A/COR1) and 
Table 1. Parameters related to the estimates of $B(r)$ from whitelight observations.

\begin{tabular}{|c|c|c|c|c|c|c|}
\hline $\begin{array}{l}\text { Time } \\
\text { (UT) }\end{array}$ & $\begin{array}{l}R_{\mathrm{sh}}{ }^{1} \\
\left(\mathrm{R}_{\odot}\right)\end{array}$ & $\begin{array}{c}R_{\mathrm{fl}}^{2} \\
\left(\mathrm{R}_{\odot}\right)\end{array}$ & $\begin{array}{c}R_{\mathrm{C}}^{3} \\
\left(\mathrm{R}_{\odot}\right)\end{array}$ & $\begin{array}{c}\Delta R \\
{\left[R_{\mathrm{sh}}-R_{\mathrm{f}}\right]} \\
\quad\left(\mathrm{R}_{\odot}\right)\end{array}$ & $\begin{array}{c}N_{\mathrm{e}} \\
{\left[4 \times N_{\mathrm{cor}}(r)\right]} \\
\left(\times 10^{5} \mathrm{~cm}^{-3}\right)\end{array}$ & $\begin{array}{c}B \\
(\mathrm{G})\end{array}$ \\
\hline \multicolumn{7}{|c|}{ STEREO-A/COR1 } \\
\hline $05: 25$ & 2.75 & 2.30 & 0.26 & 0.45 & - & - \\
\hline $05: 30$ & 3.11 & 2.50 & 0.37 & 0.61 & 9.00 & 0.30 \\
\hline \multicolumn{7}{|c|}{$\mathrm{SOHO} / \mathrm{LASCO}-\mathrm{C} 2$} \\
\hline $05: 36$ & 3.54 & 3.37 & 0.36 & 0.17 & - & - \\
\hline $05: 48$ & 4.40 & 4.02 & 0.61 & 0.38 & 2.31 & 0.12 \\
\hline
\end{tabular}

${ }^{1}$ Heliocentric distance of the CME shock.

${ }^{2}$ Heliocentric distance of the CME flux rope LE.

${ }^{3}$ Radius of curvature of the circle fitted to the flux rope in Figure 5.

$B(r) \approx 0.12 \mathrm{G}$ at $r \approx 4.40 \mathrm{R}_{\odot}(\mathrm{SOHO} / \mathrm{LASCO}-\mathrm{C} 2)$. The parameters related to the above calculations are listed in Table 1.

\subsection{Results}

Figure 6 shows the combined plot of the different estimates of $B(r)$ mentioned above. We find that the radial dependence of the data points could be described by a single power-law relation of the form $B(r)=5.7 r^{-2.6} \mathrm{G}$. For comparison, the $B(r)$ models obtained by Dulk and McLean (1978); Vršnak et al. (2002), and the $B$ values obtained by Kwon et al. (2013a; 2013b) are also overplotted in Figure 6. We find that the $B(r)$ curve obtained in this study best matches to the result of Kwon et al. (2013a).

\section{Summary}

We obtained independent estimates of $B(r)$ from the split-band type II burst observed on 23 July 2016, and the associated whitelight CME with flux rope structure. Rather than assuming any existing density model for the $N(r)$ values, we used the $N(r)$ values obtained from STEREO-A/COR1 and SOHO/LASCO$\mathrm{C} 2 \mathrm{pB}$ measurements. The $B(r)$ values, spread over the range $r \approx 2.5-4.5 \mathrm{R}_{\odot}$, could be fitted by a single power-law fit of the form $B(r)=5.7 r^{-2.6} \mathrm{G}$. The power-law index (i.e.-2.6) is in good agreement with that mentioned in the literature by different authors for variation of $B(r)$ in the outer corona: i) for example, using Faraday rotation observations of the linearly polarized carrier signals of the HELIOS spacecraft, Patzold et al. (1987) found that $B(r)$ varies as $r^{-2.7}$ in the range $r \approx 3-10 \mathrm{R}_{\odot}$; ii) recently, Mancuso and Garzelli $(2013$ ) reported $B(r)=3.76 r^{-2.29} \mathrm{G}$ in the range $r \approx 5-14 \mathrm{R}_{\odot}$ using observations of similar signals from extragalactic radio sources occulted by the solar corona. Considering $B(r)$ values at specific $r$ in the outer corona (close to the range in the present work), we find that Spangler (2005); Gopalswamy and Yashiro 


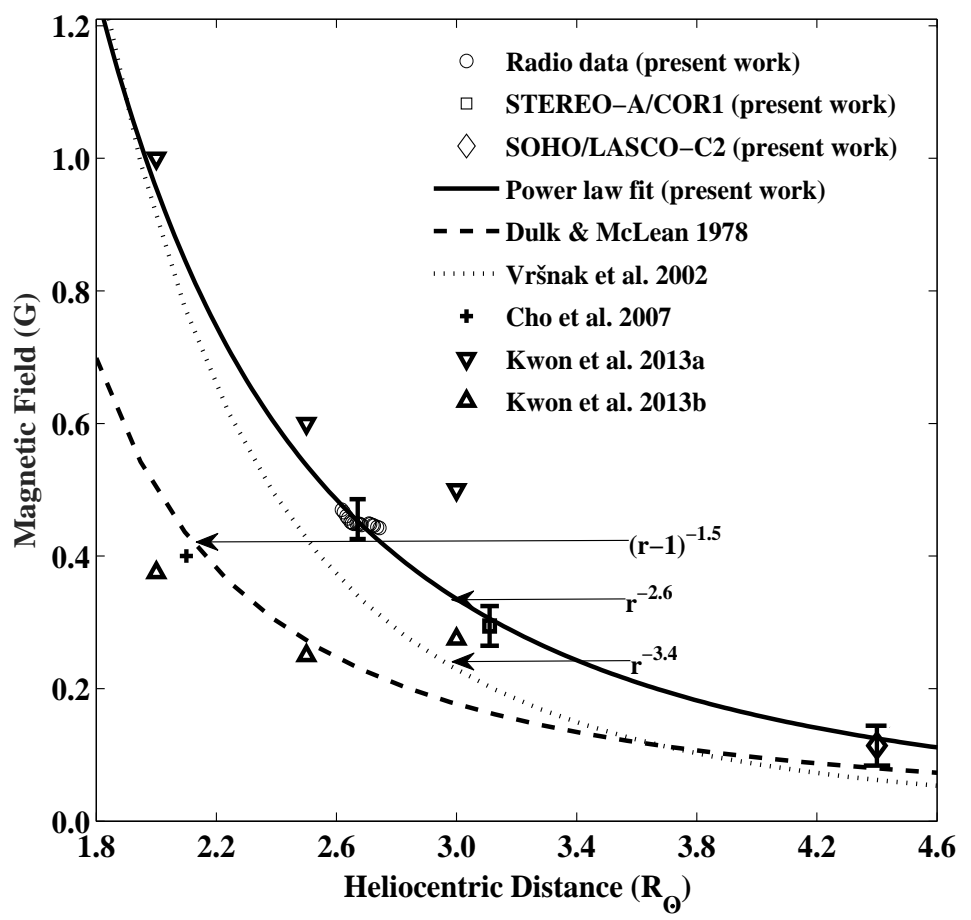

Figure 6. Estimates of $B(r)$ using band-splitting of the type II radio burst in Figure 1 and the shock stand-off technique applied to the associated whitelight CME observed with the STEREO-A/COR1 and SOHO/LASCO-C2 coronagraphs.

(2011) had estimated $B(r) \approx 39 \mathrm{mG}$ and $\approx 48 \mathrm{mG}$ at $r \approx 6.2 \mathrm{R}_{\odot}$ and $\approx 6.0 \mathrm{R}_{\odot}$, respectively. Compared to these, the power-law expression derived in the present work predicts $B(r) \approx 49 \mathrm{mG}$ and $\approx 54 \mathrm{mG}$ at the above distances. Therefore, unambiguous estimates of $B(r)$ in the middle corona can be obtained using radio and whitelight observations as described in the present work upon identifying the appropriate $N(r)$ there.

Acknowledgments We thank the staff of the Gauribidanur observatory for their help in maintenance of the antenna receiver systems and the observations. AK acknowledges $\mathrm{Mr}$. K. Hariharan and Mr. V. Mugundhan for discussions. We also thank the referee for his/her comments which helped us to present the results more clearly. The SOHO data are produced by a consortium of the Naval Research Laboratory (USA), Max-Planck-Institut fuer Aeronomie (Germany), Laboratoire d'Astronomie (France), and the University of Birmingham (UK). SOHO is a project of international cooperation between ESA and NASA. The SOHO-LASCO CME catalog is generated and maintained at the CDAW Data Center by NASA and the Catholic University of America in cooperation with the Naval Research Laboratory. The work of TJW was supported by NASA Cooperative Agreement NNG11PL10A to CUA.We also thank the referee and guest editors for their comments.

Disclosure of Potential Conflicts of interest We declare that there are no conflicts of interest for the work presented here. 


\section{References}

Anshu, K., Ramesh, R., Kathiravan, C., Gopalswamy, N.: 2017, New Evidence for a Coronal Mass Ejection-driven High Frequency Type II Burst near the Sun. Astrophys. J. 843, 10. DOI ADS

Aurass, H.: 1997, Coronal Mass Ejections and Type II Radio Bursts. In: Trottet, G. (ed.) Coronal Physics from Radio and Space Observations, Lecture Notes in Physics, Berlin Springer Verlag 483, 135. DOI ADS

Bakunin, L.M., Ledenev, V.G., Kosugi, T., McLean, D.J.: 1990, The harmonic structure of a type II burst on 12 May, 1983. Solar Phys. 129, 379. DOI ADS

Bastian, T.S., Pick, M., Kerdraon, A., Maia, D., Vourlidas, A.: 2001, The Coronal Mass Ejection of 1998 April 20: Direct Imaging at Radio Wavelengths. Astrophys. J. Lett. 558, L65. DOI ADS

Benz, A.O., Monstein, C., Meyer, H., Manoharan, P.K., Ramesh, R., Altyntsev, A., Lara, A., Paez, J., Cho, K.-S.: 2009, A World-Wide Net of Solar Radio Spectrometers: e-CALLISTO. Earth Moon and Planets 104, 277. DOI ADS

Brueckner, G.E., Howard, R.A., Koomen, M.J., Korendyke, C.M., Michels, D.J., Moses, J.D., Socker, D.G., Dere, K.P., Lamy, P.L., Llebaria, A., Bout, M.V., Schwenn, R., Simnett, G.M., Bedford, D.K., Eyles, C.J.: 1995, The Large Angle Spectroscopic Coronagraph (LASCO). Solar Phys. 162, 357. DOI ADS

Cho, K.-S., Lee, J., Gary, D.E., Moon, Y.-J., Park, Y.D.: 2007, Magnetic Field Strength in the Solar Corona from Type II Band Splitting. Astrophys. J. 665, 799. DOI ADS

Collett, E.: 1992, Polarized light. fundamentals and applications. Optical Engineering, New York: Dekker, - c1992.

Dulk, G.A., McLean, D.J.: 1978, Coronal magnetic fields. Solar Phys. 57, 279. DOI. ADS.

Dulk, G.A., Suzuki, S.: 1980, The position and polarization of Type III solar bursts. Astron. Astrophys. 88, 203. ADS

Ebenezer, E., Ramesh, R., Subramanian, K.R., SundaraRajan, M.S., Sastry, C.V.: 2001, A new digital spectrograph for observations of radio burst emission from the Sun. Astron. Astrophys. 367, 1112. DOI ADS

Ebenezer, E., Subramanian, K.R., Ramesh, R., Sundararajan, M.S., Kathiravan, C.: 2007, Gauribidanur radio array solar spectrograph (GRASS). Bulletin of the Astronomical Society of India 35, 111. ADS

Gary, D.E., Dulk, G.A., House, L.L., Illing, R., Wagner, W.J.: 1985, The Type IV burst of 1980 June 29, 0233 UT - Harmonic plasma emission? Astron. Astrophys. 152, 42. ADS

Gelfreikh, G.B.: 2004, Coronal Magnetic Field Measurements Through Bremsstrahlung Emission. In: Gary, D.E., Keller, C.U. (eds.) Astrophysics and Space Science Library, Astrophysics and Space Science Library 314, 115. DOI ADS

Gopalswamy, N.: 2006, Coronal Mass Ejections and Type II Radio Bursts. Washington DC American Geophysical Union Geophysical Monograph Series 165, 207. DOI ADS

Gopalswamy, N., Kundu, M.R.: 1990, Multiple moving magnetic structures in the solar corona. Solar Phys. 128, 377. DOI ADS

Gopalswamy, N., Yashiro, S.: 2011, The Strength and Radial Profile of the Coronal Magnetic Field from the Standoff Distance of a Coronal Mass Ejection-driven Shock. Astrophys. J. Lett. 736, L17. DOI. ADS.

Gopalswamy, N., Nitta, N., Akiyama, S., Mäkelä, P., Yashiro, S.: 2012, Coronal Magnetic Field Measurement from EUV Images Made by the Solar Dynamics Observatory. Astrophys. J. 744, 72. DOI ADS

Grognard, R.J.M., McLean, D.J.: 1973, Non-Existence of Linear Polarization in Type III Solar Bursts at $80 \mathrm{MHz}$. Solar Phys. 29, 149. DOI ADS

Hariharan, K., Ramesh, R., Kathiravan, C.: 2015, Observations of Near-Simultaneous SplitBand Solar Type-II Radio Bursts at Low Frequencies. Solar Phys. 290, 2479. DOI. ADS

Hariharan, K., Ramesh, R., Kishore, P., Kathiravan, C., Gopalswamy, N.: 2014, An Estimate of the Coronal Magnetic Field near a Solar Coronal Mass Ejection from Low-frequency Radio Observations. Astrophys. J. 795, 14. DOI ADS

Hariharan, K., Ramesh, R., Kathiravan, C., Abhilash, H.N., Rajalingam, M.: 2016a, High Dynamic Range Observations of Solar Coronal Transients at Low Radio Frequencies with a Spectro-correlator. Astrophys. J. Supp. 222, 21. DOI ADS

Hariharan, K., Ramesh, R., Kathiravan, C., Wang, T.J.: 2016b, Simultaneous Near-Sun Observations of a Moving Type IV Radio Burst and the Associated White-Light Coronal Mass Ejection. Solar Phys. 291, 1405. DOI ADS 
Howard, R.A., Moses, J.D., Vourlidas, A., Newmark, J.S., Socker, D.G., Plunkett, S.P., Korendyke, C.M., Cook, J.W., Hurley, A., Davila, J.M., Thompson, W.T., St Cyr, O.C., Mentzell, E., Mehalick, K., Lemen, J.R., Wuelser, J.P., Duncan, D.W., Tarbell, T.D., Wolfson, C.J., Moore, A., Harrison, R.A., Waltham, N.R., Lang, J., Davis, C.J., Eyles, C.J., MapsonMenard, H., Simnett, G.M., Halain, J.P., Defise, J.M., Mazy, E., Rochus, P., Mercier, R., Ravet, M.F., Delmotte, F., Auchere, F., Delaboudiniere, J.P., Bothmer, V., Deutsch, W., Wang, D., Rich, N., Cooper, S., Stephens, V., Maahs, G., Baugh, R., McMullin, D., Carter, T.: 2008, Sun Earth Connection Coronal and Heliospheric Investigation (SECCHI). Space Sci. Rev. 136, 67. DOI ADS.

Kim, R.-S., Gopalswamy, N., Moon, Y.-J., Cho, K.-S., Yashiro, S.: 2012, Magnetic Field Strength in the Upper Solar Corona Using White-light Shock Structures Surrounding Coronal Mass Ejections. Astrophys. J. 746, 118. DOI ADS

Kishore, P., Kathiravan, C., Ramesh, R., Rajalingam, M., Barve, I.V.: 2014, Gauribidanur Low-Frequency Solar Spectrograph. Solar Phys. 289, 3995. DOI ADS

Kishore, P., Ramesh, R., Kathiravan, C., Rajalingam, M.: 2015, A Low-Frequency Radio Spectropolarimeter for Observations of the Solar Corona. Solar Phys. 290, 2409. DOI ADS

Kishore, P., Ramesh, R., Hariharan, K., Kathiravan, C., Gopalswamy, N.: 2016, Constraining the Solar Coronal Magnetic Field Strength using Split-band Type II Radio Burst Observations. Astrophys. J. 832, 59. DOI ADS

Kliem, B., Krueger, A., Treumann, R.A.: 1992, Third plasma harmonic radiation in type II bursts. Solar Phys. 140, 149. DOI ADS

Kwon, R.-Y., Kramar, M., Wang, T., Ofman, L., Davila, J.M., Chae, J., Zhang, J.: 2013a, Global Coronal Seismology in the Extended Solar Corona through Fast Magnetosonic Waves Observed by STEREO SECCHI COR1. Astrophys. J. 776, 55. DOI ADS

Kwon, R.-Y., Ofman, L., Olmedo, O., Kramar, M., Davila, J.M., Thompson, B.J., Cho, K.-S.: 2013b, STEREO Observations of Fast Magnetosonic Waves in the Extended Solar Corona Associated with EIT/EUV Waves. Astrophys. J. 766, 55. DOI ADS

Lin, H., Penn, M.J., Tomczyk, S.: 2000, A New Precise Measurement of the Coronal Magnetic Field Strength. Astrophys. J. Lett. 541, L83. DOI ADS

Mancuso, S., Garzelli, M.V.: 2013, Radial profile of the inner heliospheric magnetic field as deduced from Faraday rotation observations. Astron. Astrophys. 553, A100. DOI ADS

Mancuso, S., Raymond, J.C., Kohl, J., Ko, Y.-K., Uzzo, M., Wu, R.: 2003, Plasma properties above coronal active regions inferred from SOHO/UVCS and radio spectrograph observations. Astron. Astrophys. 400, 347. DOI. ADS.

Mann, G., Classen, T., Aurass, H.: 1995, Characteristics of coronal shock waves and solar type II radio bursts. Astron. Astrophys. 295, 775. ADS

Monstein, C., Ramesh, R., Kathiravan, C.: 2007, Radio spectrum measurements at the Gauribidanur observatory. Bulletin of the Astronomical Society of India 35, 473. ADS.

Nelson, G.J., Melrose, D.B.: 1985, In: McLean, D.J., Labrum, N.R. (eds.) Type II bursts, 333. ADS

Patzold, M., Bird, M.K., Volland, H., Levy, G.S., Seidel, B.L., Stelzried, C.T.: 1987, The mean coronal magnetic field determined from HELIOS Faraday rotation measurements. Solar Phys. 109, 91. DOI. ADS.

Poomvises, W., Gopalswamy, N., Yashiro, S., Kwon, R.-Y., Olmedo, O.: 2012, Determination of the Heliospheric Radial Magnetic Field from the Standoff Distance of a CME-driven Shock Observed by the STEREO Spacecraft. Astrophys. J. 758, 118. DOI ADS.

Ramesh, R.: 2011, Low frequency solar radio astronomy at the Indian Institute of Astrophysics (IIA). In: Astronomical Society of India Conference Series, Astronomical Society of India Conference Series 2. ADS

Ramesh, R., Kathiravan, C., Narayanan, A.S.: 2011, Low-frequency Observations of Polarized Emission from Long-lived Non-thermal Radio Sources in the Solar Corona. Astrophys. J. 734, 39. DOI ADS

Ramesh, R., Kathiravan, C., Sastry, C.V.: 2003, Metric Radio Observations of the Evolution of a "Halo" Coronal Mass Ejection Close to the Sun. Astrophys. J. Lett. 591, L163. DOI] ADS

Ramesh, R., Kathiravan, C., Sastry, C.V.: 2010, Estimation of Magnetic Field in the Solar Coronal Streamers Through Low Frequency Radio Observations. Astrophys. J. 711, 1029. DOI. ADS

Ramesh, R., Kathiravan, C., Satya Narayanan, A.: 2004, Seismology of the solar corona through observations of metric type IV radio burst emission. Asian Journal of Physics 13, 277. ADS 
Ramesh, R., Kathiravan, C., Narayanan, A.S., Ebenezer, E.: 2003, Metric observations of transient, quasi-periodic radio emission from the solar corona in association with a "halo" CME and an "EIT wave" event. Astron. Astrophys. 400, 753. DOI ADS

Ramesh, R., Kathiravan, C., Barve, I.V., Beeharry, G.K., Rajasekara, G.N.: 2010a, Radio Observations of Weak Energy Releases in the Solar Corona. Astrophys. J. 719, L41. DOI ADS

Ramesh, R., Kathiravan, C., Kartha, S.S., Gopalswamy, N.: 2010b, Radioheliograph Observations of Metric Type II Bursts and the Kinematics of Coronal Mass Ejections. Astrophys. J. 712, 188. DOI ADS

Ramesh, R., Anna Lakshmi, M., Kathiravan, C., Gopalswamy, N., Umapathy, S.: 2012, The Location of Solar Metric Type II Radio Bursts with Respect to the Associated Coronal Mass Ejections. Astrophys. J. 752, 107. DOI ADS

Ramesh, R., Kishore, P., Mulay, S.M., Barve, I.V., Kathiravan, C., Wang, T.J.: 2013, Lowfrequency Observations of Drifting, Non-thermal Continuum Radio Emission Associated with the Solar Coronal Mass Ejections. Astrophys. J. 778, 30. DOI ADS

Roberts, J.A.: 1959, Solar Radio Bursts of Spectral Type II. Australian Journal of Physics 12, 327. DOI ADS

Ryabov, B.: 2004, Coronal Magnetic Field Measurements Through Quasi-Transverse Propagation. In: Gary, D.E., Keller, C.U. (eds.) Astrophysics and Space Science Library, Astrophysics and Space Science Library 314, 135. DOI ADS

Saito, K., Poland, A.I., Munro, R.H.: 1977, A study of the background corona near solar minimum. Solar Phys. 55, 121. DOI ADS

Sasikumar Raja, K., Ramesh, R.: 2013, Low-frequency Observations of Transient Quasiperiodic Radio Emission from the Solar Atmosphere. Astrophys. J. 775, 38. DOI ADS.

Sasikumar Raja, K., Ramesh, R., Hariharan, K., Kathiravan, C., Wang, T.J.: 2014, An Estimate of the Magnetic Field Strength Associated with a Solar Coronal Mass Ejection from Low Frequency Radio Observations. Astrophys. J. 796, 56. DOI ADS

Sastry, C.V.: 2009, Polarization of the Thermal Radio Emission from Outer Solar Corona. Astrophys. J. 697, 1934. DOI. ADS

Smerd, S.F., Sheridan, K.V., Stewart, R.T.: 1975, Split-Band Structure in Type II Radio Bursts from the Sun. Astrophys. Lett. 16, 23. ADS.

Smerd, S.F., Wild, J.P., Sheridan, K.V.: 1962, On the Relative Position and Origin of Harmonics in the Spectra of Solar Radio Bursts of Spectral Types II and III. Australian Journal of Physics 15, 180. DOI ADS

Spangler, S.R.: 2005, The Strength and Structure of the Coronal Magnetic Field. Space Sci. Rev. 121, 189. DOI ADS

Tun, S.D., Vourlidas, A.: 2013, Derivation of the Magnetic Field in a Coronal Mass Ejection Core via Multi-frequency Radio Imaging. Astrophys. J. 766, 130. DOI. ADS.

van de Hulst, H.C.: 1950, The electron density of the solar corona. Bulletin of the Astronomical Institutes of the Netherlands 11, 135. ADS

Vršnak, B.: 2001, Solar flares and coronal shock waves. J. Geophys. Res. 106, 25291. DOI ADS

Vršnak, B., Magdalenić, J., Aurass, H., Mann, G.: 2002, Band-splitting of coronal and interplanetary type II bursts. II. Coronal magnetic field and Alfvén velocity. Astron. Astrophys. 396, 673. DOI ADS

Wang, T., Davila, J.M.: 2014, Validation of Spherically Symmetric Inversion by Use of a Tomographically Reconstructed Three-Dimensional Electron Density of the Solar Corona. Solar Phys. 289, 3723. DOI ADS

White, S.M.: 2004, Coronal Magnetic Field Measurements Through Gyroresonance Emission. In: Gary, D.E., Keller, C.U. (eds.) Astrophysics and Space Science Library, Astrophysics and Space Science Library 314, 89. DOI. ADS.

Wild, J.P., Smerd, S.F., Weiss, A.A.: 1963, Solar Bursts. Ann. Rev. Astron. Astrophys. 1, 291. DOI ADS

Zlotnik, E.Y., Klassen, A., Klein, K.-L., Aurass, H., Mann, G.: 1998, Third harmonic plasma emission in solar type II radio bursts. Astron. Astrophys. 331, 1087. ADS 